\title{
Eltern stützen, Kinder schützen
}

\section{Nach den Gesetzesänderungen: Wie zukunftsfest ist das Kinder- und Jugendhilferecht?}

\author{
Johannes Münder
}

\begin{abstract}
Die Kinder-und Jugendhilfe hatte in den letzten Jahren eine Reihe von Änderungen ibrer gesetzlichen Grundlagen zu verarbeiten. Die Föderalismusreform stellt nun die erreichten Standards und die bisherige Organisation behördlicher Kinder- und Jugendhilfe infrage.
\end{abstract}

In seinen über 15 Jahren seit dem In-KraftTreten des SGB VIII - Kinder- und Jugendhilfe am 1. Januar 1991 (1) hat das SGB VIII zahlreiche Veränderungen erfahren (2), diese sind natürlich von unterschiedlicher inhaltlicher Bedeutung (3).

Schwerpunkte gesetzlicher Änderungen in den letzten Jahren waren das Gesetz zum qualitätsorientierten und bedarfsgerechten Ausbau der Tagesbetreuung für Kinder (Tagesbetreuungsausbaugesetz TAG) (4) und das Gesetz zur Weiterentwicklung der Kinder- und Jugendhilfe (Kinder- und Jugendhilfeentwicklungsgesetz - KICK) (5). Nicht unerhebliche, wenn auch punktuelle Auswirkungen brachten andere Gesetze: Erwähnt sei hier das Gesetz über den Aufenthalt, die Erwerbstätigkeit und die Integration von Ausländern im Bundesgebiet (Aufenthaltsgesetz - AufenthG) (6) und das 4. Gesetz für moderne Dienstleistungen am Arbeitsmarkt (7).

Derzeit lässt sich noch nicht absehen, ob es nicht noch langfristig möglicherweise gravierende Änderungen geben wird aufgrund von Verfassungsänderun-

Prof. Dr. jur. Johannes Münder lehrt am Institut für Gesellschaftswissenschaften und historisch-politische Bildung an der Technischen Universität Berlin mit dem Schwerpunkt Sozial- und Zivilrecht. Kürzlich erschien die von ihm herausgegebene Neuauflage des Frankfurter Kommentars zum Kinderund Jugendhilfegesetz. Johannes Münder ist zudem Vorsitzender des SOS-Kinderdorf e. V.

E-Mail johannes.muender@tu-berlin.de gen: Im Zusammenhang mit der »Neuordnung der bundesstaatlichen Ordnung ", der so genannten Föderalismusdiskussion konnte beim ersten Anlauf dieses Vorhabens verhindert werden, dass die Forderung der Länder nach einer ausschließlichen Gesetzgebungskompetenz für »ergänzende öffentliche Leistungen im Bereich der Bildung und Erziehung ", die sich wesentlich auf das SGB VIII bezog, realisiert wurde (8).

Nach gegenwärtigem Stand aber ist es durchaus möglich, dass die in Art. 74 Abs. 1 Nr. 7 GG geregelte »Fürsorge« als Gegenstand der konkurrierenden Gesetzgebung entsprechend dem Entwurf zu Art. 72 Abs. 2 GG nur dann unter die bundesgesetzliche Kompetenz fällt, wenn und soweit »die Herstellung gleichwertiger Lebensverhältnisse im Bundesgebiet oder die Wahrung der Rechts- oder Wirtschaftseinheit im gesamtstaatlichen Interesse eine bundesgesetzliche Regelung erforderlich macht «. Weitere mögliche Auswirkungen ergeben sich aus Art. 84 GG. Danach kann der Bund nicht mehr die Einrichtung von Behörden vorschreiben, wenn die Länder entsprechende Bundesgesetze ausführen - was beim SGB VIII der Fall ist. Das bedeutet insbesondere, dass die Errichtung von Jugendämtern nicht mehr vorgeschrieben werden kann.

Nach alledem: Ist die Kinder- und Jugendhilfe zukunftsfest? Die Beantwortung dieser Frage hängt davon ab, was man als Aufgabe der Kinder- und Jugendhilfe für die Bewältigung der anstehenden Zukunft betrachtet. Die Problemlagen von Kindern, Jugendlichen und Familien in unserer Gesellschaft sind vielfältig.

Aber: Ist Aufgabe der Kinder- und Jugendhilfe hier problemlösend tätig zu sein? Kann sie das überhaupt allein? Und ist sie möglicherweise sogar zu Unrecht gefordert, wenn man etwa an das Stichwort der demografischen Entwicklung denkt?
Nur wer Allmachtsfantasien hinsichtlich der Kinder- und Jugendhilfe hegt, wird die Kinder- und Jugendhilfe, die Soziale Arbeit, die Sozialpädagogik zum Retter zumindest der Welt von Kindern und Jugendlichen machen wollen. Bei einer nüchternen Betrachtung der Wirkund Reichweite von Kinder- und Jugendhilfe wird man bescheidender sein: Sie kann ihren Beitrag dazu leisten und sie wird an manchen Stellen in besonderer Weise dazu gefordert sein.

Mit dem Tagesbetreuungsausbaugesetz und dem Kinder- und Jugendhilfeentwicklungsgesetz ist sie an zwei wichtigen Stellen mit den Lebenslagen von Kindern und Jugendlichen befasst:

- Das Tagesbetreuungsausbaugesetz dient in erster Linie dem Ausbau und der Qualifizierung von Tageseinrichtungen und Kindertagespflege. Damit nimmt Kinder- und Jugendhilfe ihren (sicher auch hier: nicht alleinigen) Bildungsauftrag an und versucht in ihrem Bereich das zu tun, was notwendig ist: eine verlässliche, inhaltlich auf die Förderung und Bildung ausgerichtete, Tagesbetreuung von Kindern.

- Das Kinder- und Jugendhilfeentwicklungsgesetz hat seine inhaltlichen Schwerpunkte im Kinderschutz (insbesondere $\int 8$ a SGB VIII) und in der Steuerungsverantwortung des Jugendamtes (insbesondere $\mathbb{S} 36 \mathrm{a}$ SGB VIII). Und wesentlicher Inhalt der Steuerungsverantwortung ist in der Tat die Verantwortung und nicht »Macht «, etwa gar in dem Sinne verstanden, dass man nun Ansprüche von Kindern, Jugendlichen, Eltern leichter abwehren kann. Sondern in dem Sinne, dass sich Jugendhilfe darüber im Klaren sein muss, dass es ihre eigene, originäre Aufgabe ist, sich darum zu kümmern, welche individuellen Hilfen erforderlich und notwendig sind.

Mit der Betonung des Kinderschutzes wird eine eigentlich uralte Aufgabe (möglicherweise: wieder) klar: dass Kinder- 
und Jugendhilfe es nicht nur mit der glänzenden Vorderseite von Leistungen und Angeboten zu tun hat, sondern auch mit der schattigen Rückseite von Eingriffen, harten Interventionen. Dass es nicht nur um »Eltern stützen «, sondern auch um »Kinder schützen « geht.

Dieses alles sind und können in Kürze nur allgemeine, globale Hinweise sein. Im Detail sind die Probleme schwierig, komplex, unterschiedlich. Neben der erforderlichen sozialpädagogischen Kompetenz wird man sich auch immer wieder mit den rechtlichen Rahmenbedingungen befassen müssen.
Oktober 2005 auf 25 Änderungen; vgl. Wiesner/Wiesner SGB VIII Einleitung Rz. 8 ff.

(3) Im Folgenden konzentriere ich mich auf das Leistungsrecht. In der vorletzten Legislaturperiode stand im Schwerpunkt von Gesetzesänderungen das Leistungserbringungsrecht, insbesondere mit der ab dem 1. Januar 1999 in den $\mathbb{S} \mathbb{S} 78 \mathrm{a}$ ff. SGB VIII neu geregelten Vereinbarungen über Leistungsangebote, Entgelte und Qualitätsentwicklung, die dem gemäß in den - gebundenen - Kommentaren der Vorauflagen im Mittelpunkt stan-

\section{»In der Kinder-und Jugendhilfe geht es - leider - nicht nur um Leistungen, sondern auch um Eingriffe"}

Im Gegensatz zu anderen Sozialleistungsbereichen wird in der Kinder- und Jugendhilfe von den Betroffenen wenig geklagt, sie haben in der Regel keinen $\mathrm{Zu}-$ gang zum Rechtssystem. Deswegen ist es hier mehr als in anderen Sozialleistungsbereichen Aufgabe der professionellen Akteure, bereits bei ihrem fachlichen Handeln die rechtlichen Vorgaben und Vorstellungen zu berücksichtigen.

Für die Arbeit an juristischen Materien - wie etwa bei Kommentierungen zum SGB VIII - bedeutet dies eine entsprechende sozialpädagogische, sozial- und humanwissenschaftliche Ausrichtung. Die entsprechenden Neuauflagen von Kommentaren sind deswegen nicht nur an ihrer rechtsdogmatischen Sorgfalt, sondern an ihrer sozialpädagogischen und sozialwissenschaftlichen Ausrichtung zu messen.

\section{Anmerkungen}

(1) Bekanntermaßen ist das SGB VIII im Rahmen des 2. Staatsvertrages zwischen BRD und DDR - Einigungsvertrag - auf das Beitrittsgebiet übergeleitet worden und sofort in Kraft gesetzt und dem gemäß im Beitrittsgebiet am 3. Oktober 1990 in Kraft getreten.

(2) Wiesner hat die Änderungen des Gesetzes gezählt und kommt bis zum den, vgl. z. B. Frankfurter Kommentar zum SGB VIII: Kinder- und Jugendhilfe, 4. Auflage. Zu den entsprechenden Änderungen im Leistungsrecht in der letzten Legislaturperiode vgl. nunmehr Münder u. a. Münder u. a. Frankfurter Kommentar zum SGB VIII: Kinder- und Jugendhilfe, 5. vollständig überarbeitete Auflage, Weinheim/München 2006.

(4) Vom 27. Dezember 2004 - BGBl. I, 3852.

(5) Vom 8. September 2005 - BGBl. I, 2729.

(6) Vom 30. Juli 2004 - BGBl. I, 1950, das das vom Bundesverfassungsgericht als verfassungswidrig aufgehobene (BVerfG 18.12.2002 - 2 BvF 1/02 - BGBl. I 2002, 126) Zuwanderungsgesetz ersetzte, das nicht unerhebliche Auswirkungen auf die in $\$ 6$ SGB VIII vorgenommene Regelung unter dem Stichwort des Geltungsbereichs für Ausländer hat.

(7) Vom 24. Dezember 2003 - BGBl. I, 2954, wobei der spezielle Nachrang des $\mathbb{} 13$ SGB VIII gegenüber dem SGB II nicht selbst durch dieses Gesetz vorgenommen wurde, sondern erst - in korrigierender Weise - bewirkt wurde durch das Gesetz zur optionalen Trägerschaft von Kommunen nach dem Zweiten Sozialgesetzbuch vom 30. Juni 2004 - BGBl. I, 2014.
(8) Dazu trugen wesentlich entsprechende Interventionen von Verbänden und Organisationen aus dem Bereich der Kinder- und Jugendhilfe sowie der Appell namhafter Einzelpersönlichkeiten bei.

\section{Literatur}

Johannes Münder u. a.: Frankfurter Kommentar zum SGB VIII: Kinderund Jugendhilfe. Juventa Verlag, Weinheim 2006. 1.204 Seiten. 74,- Euro. ISBN 3-7799-1882-X. 